\title{
Estimation of the Deformation Temporal Evolution Using Airborne Differential SAR Interferometry
}

\author{
Pau Prats*§, Andreas Reigber ${ }^{\dagger}$, Jordi J. Mallorqui*, Pablo Blanco* and Alberto Moreira ${ }^{\ddagger}$ \\ ${ }^{*}$ Universitat Politecnica de Catalunya (UPC), Dept. of Signal Theory and Communications, Spain \\ Email: \{pprats,mallorqui,pblanco\}@tsc.upc.edu \\ ${ }^{\dagger}$ Berlin University of Technology (TUB), Computer Vision and Remote Sensing Group, Germany \\ Email: anderl@cs.tu-berlin.de \\ ${ }_{\ddagger}$ German Aerospace Center, Microwave and Radar Institute, Germany \\ Email: alberto.moreira@dlr.de \\ §Universitat Autònoma de Barcelona (UAB), Dept. of Telecommunications and Systems Engineering, Spain
}

\begin{abstract}
This paper presents airborne DInSAR results using a stack of 14 images, which were acquired by the Experimental SAR (E-SAR) system of the German Aerospace Center (DLR) during a time span of only three hours and fifteen minutes. An advanced differential technique is used to retrieve the error in the digital elevation model (DEM) and the temporal evolution of the deformation for every coherent pixel in the image. Furthermore, some modifications in the differential processing chain are included to deal with the existence of the so-called residual motion errors, which play a similar role as atmospheric artifacts in the spaceborne case. The detected deformation of a corner reflector and of some agricultural fields allows to validate the proposed techniques to measure deformation phenomena with an airborne platform.
\end{abstract}

\section{INTRODUCTION}

Differential synthetic aperture radar interferometry (DInSAR) has become a powerful tool to measure deformation phenomena at a large scale. Very high accuracy can be attained by exploiting the coherent nature of SAR systems to obtain a precision that is in the order of a fraction of the wavelength. Differential SAR interferometry using a spaceborne platform is already a quite established technique, since the stable trajectory of the satellite ensures the SAR processor will focus the data without introducing undesired artifacts. Also, the fact that large stacks of images are available has been of great help to develop several algorithms. However, the airborne case is almost the opposite. First, there exist only very few differential data sets with in-situ measurements to be able to validate results. But more importantly, the data processing becomes a challenge itself since it is subject to the limitations imposed by motion compensation (MoCo). The fact that the platform does not follow an ideally rectilinear trajectory arises several drawbacks that must be considered if accuracy is a priority. However, the advantages an airborne platform offers are quite appealing: flexibility in sense of spatial resolution, used wavelength, and data acquisition. Furthermore, the atmosphere has little or no impact, depending on the used wavelength and flight altitude, and the costs of upgrading the hardware of an airborne system are insignificant compared to those necessary to launch a new satellite with improved performances.
The limitations existing in airborne repeat-pass interferometry are mainly two: the presence of residual motion errors (RME), and the fact that some kind of reference height must be assumed during motion compensation. Section II-A analyzes them, while Section II-B comments the processing approach to focus the images and circumvent the limitations imposed by motion compensation when working with airborne repeatpass interferometric data. Section III presents the modifications that have been implemented to adapt the selected advanced differential technique to the airborne case. Finally, Section IV presents some results with data acquired by the E-SAR system of DLR.

\section{Airborne CONSIDERATions}

\section{A. Limitations with airborne platforms}

The first limitation in airborne repeat-pass interferometry arises due to the assumption of a reference height during motion compensation. This assumption implies that the phase history of targets at a height different than the reference one is not properly corrected, inducing mainly phase errors and displacement of the impulse response. Given both master and slave tracks are uncorrelated, these effects turn into important phase and coregistration errors in the generated interferogram. Note that in the single-pass case, these errors can be neglected as the trajectories are correlated. In [1], a MoCo algorithm capable of accommodating topography variations accurately is proposed. This correction is applied after conventional $2^{\text {nd }}$ order MoCo [2] and before azimuth compression, consisting in a range-dependent phase multiply using sub-apertures, based on the well known time-frequency relation of SAR systems. In this way, the topography variations within the antenna footprint can be taken into account. With this efficient approach is then possible to retrieve an interferogram of high quality, where phase and coregistration errors are minimized.

The second and most challenging limitation is the existence of residual motion errors, i.e. inaccuracies in the navigation data, which can lead again to important phase and coregistration errors in the final interferogram. Current navigation systems can reach accuracies around $5-10 \mathrm{~cm}$, depending particulary on GPS baseline as well as other factors. Note 
for example that a $2 \mathrm{~cm}$ error implies a $60^{\circ}$ phase error at L-band. In [3] a multi-squint technique based on spectral diversity is proposed to efficiently estimate residual motion errors affecting a given interferogram. This technique relates the azimuthal coregistration error with the derivative of RME. Once RME are estimated, the original navigation data are then corrected so that the raw data can be re-processed accurately.

\section{B. Data Processing}

The fact that both limitations have similar effects implies several changes in the processing chain are required: first, standard motion compensation has to be performed adaptively to the local topography, and then multi-squint spectral diversity estimation of RME can be applied. Therefore, the approach presented in [4], which includes the commented algorithms [1], [3], has been used to process the data.

Since in differential interferometry there are more than two images, the reference trajectories of all acquisitions must be imposed to be parallel to obtain the maximum benefit. Doing so, all the images will appear aligned along azimuth after the SAR focusing operation. A first iteration using a large bandwidth allows a good estimation of residual motion errors with multi-squint. After updating the tracks, data are again reprocessed, this time with a smaller bandwidth. Since after the correction of residual motion errors the individual images still have a mixture of the individual residual motion errors inside, this implies both master and slave images must be re-processed for each interferogram. Only if the same master image is used for all interferograms, can the re-processing be applied only to the slave images, provided the full correction of residual motion errors is applied to them. Note that this is not the case in the results of Section IV, where several images are used as master.

It is interesting to note that after the correction of residual motion errors the individual RME of each image remains unknown. This implies that, although a given pair of images forming an interferogram are aligned along azimuth, they might not be aligned to other images, resulting in a small misalignment between interferograms. However, in practice this can be neglected, for the azimuth coregistration offset due to RME is generally smaller than an azimuth resolution cell ( $\mathrm{RME}$ are smaller than $\pm 2 \mathrm{~cm}$ in line of sight (LOS) in the current data set). Furthermore, if multilooking is applied to each interferogram, as it is the case for the presented results (a multilook of $4 \times 4$ pixels has been applied), this misregistration can be completely neglected.

\section{Height Sensitivity}

It is already established in literature that the flattened phase is proportional to the real baseline, i.e. to the real antenna positions (not to the reference ones imposed during MoCo), and to the difference between the true topographic height and the one used as reference during motion compensation [5]. However, since now an external DEM is being used during motion compensation, the residual phase, i.e. the phase that remains after subtracting the synthetic phase computed with the external DEM used during MoCo, is sensitive to the real baseline and to the DEM error, i.e.

$$
\phi_{\text {residual }} \approx k_{\mathrm{z}}^{\text {real }} h_{\text {error }},
$$

with

$$
k_{\mathrm{z}}^{\text {real }}=-\frac{4 \pi}{\lambda} \frac{B_{\perp}^{\text {real }}}{r \sin \theta},
$$

$B_{\perp}^{\text {real }}$ being the real perpendicular baseline, $\lambda$ the used wavelength, $r$ the range distance, and $\theta$ the off-nadir look angle. Hence, it is straightforward to use this information to correct the original DEM by just scaling the unwrapped residual phase using the real baseline. This solution is important since it is more accurate than using the reference baseline in, for example, differential interferometry. Note also that the larger the baseline gets with respect to the platform deviations, the more similar are the reference and real baselines.

\section{ADVANCED AIRBORNE DINSAR}

The differential technique presented by Berardino and et al. [6] has been selected to process the data of Section IV. It has been preferred as it is quite straightforward to implement once the interferograms have been unwrapped, which is usually the critical step in differential interferometry. It turns out that the data to be presented have very good coherence, making phase unwrapping trivial in this case.

After phase unwrapping and calibration of all residual interferograms, all the pixels that accomplish a given criterium, e.g. a mean coherence through all interferograms larger than a certain threshold, are selected. Then, a least-squares (LS) estimation is performed for each selected pixel, which considers a DEM error and a mean deformation velocity. As noted before, in the airborne case the real baseline must be used instead of the reference one. After subtracting the estimated DEM error and mean deformation velocity to each interferogram, now in theory only the non-linear deformation should remain. However, residual motion errors might still persist in the images, similarly as it happens in the spaceborne case with atmospheric artifacts. It turns out that in the airborne case the geometry of residual motion errors is known; they are the projection in LOS of the individual horizontal and vertical residual motion errors

$$
\epsilon_{\mathrm{los}}(x, r)=\epsilon_{y}(x) \sin \theta(r)-\epsilon_{z}(x) \cos \theta(r),
$$

where $\epsilon$ represents residual motion errors. Hence, a better approach than using a large averaging window in the spatial domain (a low-pass filter is applied in the spaceborne case) is to apply a LS estimation in a similar way as in [3] to each differential interferogram in order to estimate persistent residual motion errors, i.e.

$$
\epsilon_{\mathrm{xy}}=\left(\mathbf{A}^{\mathrm{T}} \mathbf{A}\right)^{-1} \mathbf{A} \epsilon_{\mathrm{los}}
$$

where the matrix $\mathbf{A}$ considers the model in (3), $\epsilon_{\mathrm{xy}}$ is the desired estimates vector, and $\epsilon_{\text {los }}$ is the phase value of the pixels included in the estimation. Since all pixels are assumed to have an acceptable coherence level, no weighting is applied in the LS estimation. Two approaches are proposed: 

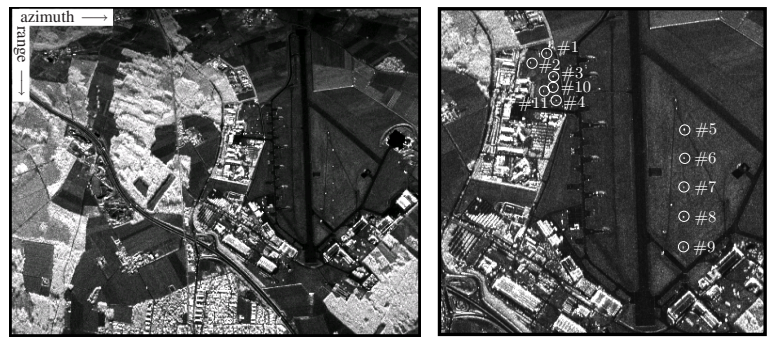

Fig. 1. (left) Reflectivity image of the scene under study and (right) detail of corner reflectors.
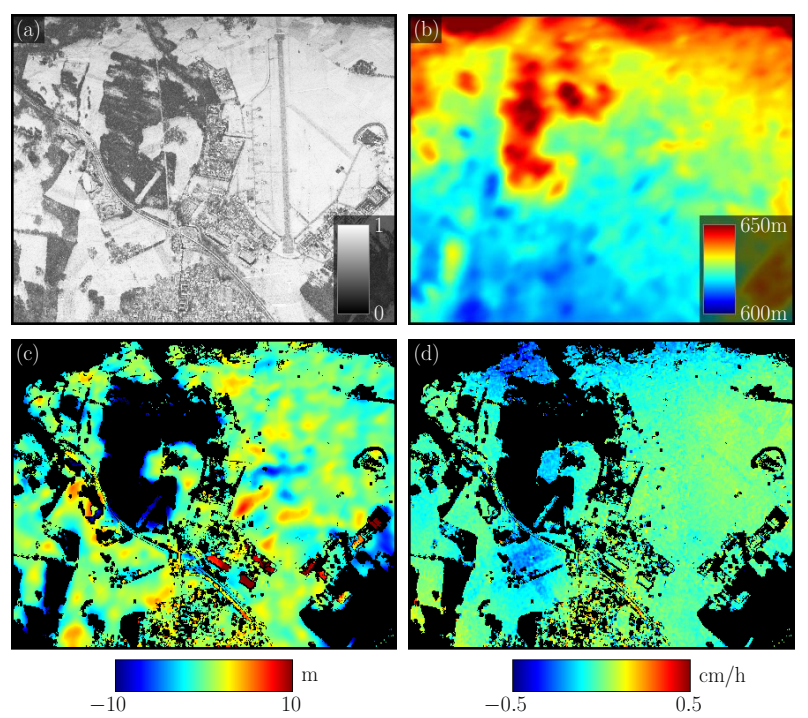

Fig. 2. (a) Mean coherence, (b) SRTM DEM used during motion compensation, (c) retrieved DEM error and (d) retrieved mean deformation velocity. Masked values in (c) and (d) in black.

- Low order filter: the LS only considers constant and linear terms of residual motion errors (see [3] for details). Therefore, it is assumed that the multi-squint has been able to perform quite accurately, but constant and linear terms might have not been properly retrieved.

- High order filter: the LS is applied to each range line in order to estimate any possible residual motion error. Obviously, in this case the probability to filter out deformation information is higher.

Once residual motion errors are estimated in each differential interferogram, they are subtracted. An SVD is applied to the mean phase velocity between time-adjacent acquisitions (see [6] for details) in order to obtain the deformation in each image. Finally, a low-pass filter in time-domain can be applied to further reduce residual motion errors.

\section{RESUlTS}

A total of 14 images were acquired by the E-SAR system of DLR during a time span of only three hours and fifteen minutes at L-band (15min between each acquisition), which ensured a good coherence through all the images. The data acquisition took place on May $3^{\text {rd }} 1998$ in order to carry
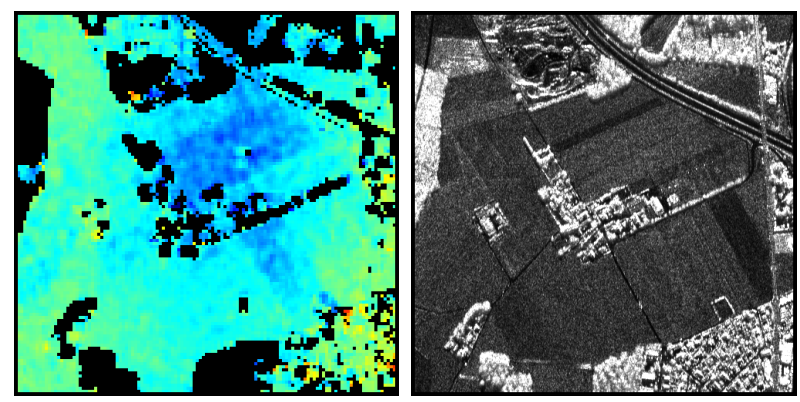

Fig. 3. (left) Detail of mean deformation velocity map with the same scale as Fig. 2(d) and (right) reflectivity image of the same area.

out the first tomographic experiment with a SAR system [7]. This same data set has been used to analyze the performance and limitations of advanced DInSAR techniques when working with airborne data. With 14 images, up to 91 interferograms can be generated. However, a maximum baseline of $90 \mathrm{~m}$ has been imposed in order to reduce the impact of some approximations during the differential processing, resulting in a total number of 51 interferograms. Fig. 1 shows the reflectivity image of the observed scene together with a detail of the corner reflectors, while Fig. 2(a) shows the mean coherence of all 51 interferograms and Fig. 2(b) the SRTM DEM used during motion compensation.

All residual interferograms have been calibrated using corner reflector number 6 (see Fig. 1). After applying a LS estimation to each pixel, the DEM error and the mean deformation velocity maps are obtained. Fig. 2(c) shows the former, where it can be noted that the height of some buildings has been properly retrieved, as one of the interferograms with a smaller baseline supported the phase unwrapping of the others. On the other hand, Fig. 2(d) depicts the mean deformation velocity map, which shows a quite stable image. Some effects can be observed at near range that the authors attribute to some approximations applied during the DInSAR processing.

Also, some deformation is observed in corner reflector number 11, which was indeed moved in purpose during the data take. Unexpectedly, some rectangular shapes on the bottom show quite clearly and they do not seem to be a processing artifact. In fact, if this image is compared to the reflectivity map as depicted in Fig. 3, it can be noted how the shapes correlate well with some agricultural fields. Hence, some deformation is indeed being observed. Since a negative displacement in LOS represents a rise of the phase center, a plausible explanation is the soil moisture increased during the data take maybe due to defrost of the vegetation or irrigation (the experiment started at 11:00), reducing in this way the penetration depth of the electromagnetic waves.

After subtracting the DEM error and the mean deformation velocity, one of the two filtering approaches commented in Section III should be applied to each interferogram. The high order filter resulted in a better performance. The authors believe this is because the measurement of the forward velocity was not accurate enough, overestimating in this case the 

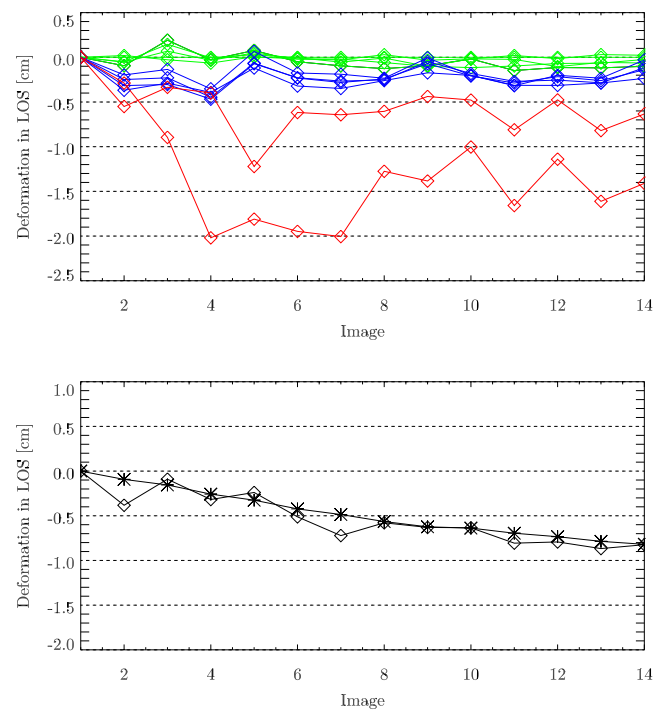

Fig. 4. Deformation evolution in LOS for (top) all corner reflectors without filter in time domain and (bottom) a pixel in the agricultural fields showing some deformation with (stars) and without (diamonds) the low-pass filter in time domain

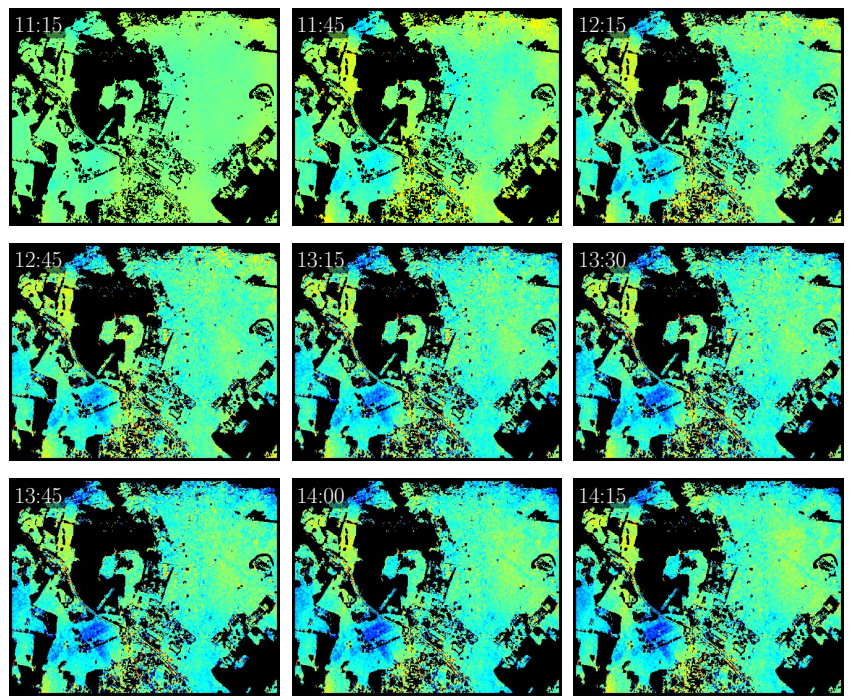

Fig. 5. Deformation evolution in LOS, with the local time of each image acquisition on the top-left corner. Acquisition started at 11:00. Scale between (blue) $-1 \mathrm{~cm}$ and (red) $1 \mathrm{~cm}$.

estimated residual motion errors with multi-squint. On the other hand, some of the acquisitions have quite large deviations from the reference track $(>15 \mathrm{~m})$, increasing the error in some approximations carried out during the processing. Once the persistent residual motion errors have been removed, the deformation of the temporal evolution along all 14 images can be retrieved. Finally, a low-pass filter in time-domain with a discrete cut-off frequency of 0.1 is applied to obtain the final deformation evolution.

Fig. 4 shows the deformation evolution for the corner reflectors and a pixel in the agricultural fields showing some deformation. In the former case, the green lines correspond to the corners next to the runway, the blues to the fixed ones on the left (corners 1 to 4 ) and the reds to the mobile ones. All blue corners show a similar behavior that obviously comes from uncorrected residual motion errors. On the other hand, the red mobile corner showing a larger deformation was the one moved on purpose (number 11), but unfortunately these results cannot be validated since the in-situ measurements are not available. On the other hand, the deformation in the agricultural field shows quite linear. As a final result, Fig. 5 shows the deformation at some time instants (note that the acquisition started at 11:00 and finished at 14:15, with a fifteen minutes interval between acquisitions), where the deformation of the fields can be clearly observed. In this case both the high order filter and the low-pass filter in time domain have been applied.

\section{CONCLUSION}

This paper has shown the potential of airborne platforms to retrieve differential interferometric products, for the first time presenting results with a large stack of images and advanced DInSAR techniques. Besides the detection of a corner reflector that was moved on purpose, it has been possible with L-band data to detect some deformation of around half a centimeter in agricultural fields, probably due to an increase of soil moisture. The good coherence of the data set turns into an optimum environment to remove residual motion errors, which represent the main accuracy limitation in current airborne systems. However, some aspects in the processing can still be improved and need some more research. Ideally, a proper validation of the proposed techniques should be carried out by performing a campaign over a scenario with real deformation and together with in-situ measurements.

\section{ACKNOWLEDGMENTS}

This work has been financed by the Spanish MCYT and EU FEDER funds under project TEC2005-06863-C02-01, by the Catalan Commission for Research (CIRIT), and by the MEC/DAAD under the Spanish-German Integrated Action HA2003-113/D-03-40325.

\section{REFERENCES}

[1] P. Prats, A. Reigber, and J. J. Mallorqui, "Topography-dependent motion compensation for repeat-pass interferometric SAR systems," vol. 2, no. 2, pp. 206-210, Apr. 2005.

[2] A. Moreira, J. Mittermayer, and R. Scheiber, "Extended chirp scaling algorithm for air- and spaceborne SAR data processing in stripmap and scanSAR imaging modes," IEEE Trans. Geosci. Remote Sensing, vol. 34 no. 5, pp. 1123-1136, Sept. 1996.

[3] A. Reigber, P. Prats, and J. J. Mallorqui, "Refined estimation of timevarying baseline errors in airborne SAR interferometry," vol. 3, no. 1, pp. 145-149, Jan. 2006.

[4] P. Prats, A. Reigber, and J. J. Mallorqui, "Retrieval of high-quality interferometric SAR products with airborne repeat-pass systems," in Proc. IEEE International Geoscience and Remote Sensing Symposium (IGARSS'05), vol. 7, Seoul, Korea, July 25-29, 2005, pp. 4583-4585.

[5] D. R. Stevens, I. G. Cumming, and A. L. Gray, "Options for airborne interferometric SAR motion compensation," IEEE Trans. Geosci. Remote Sensing, vol. 33, no. 2, pp. 409-420, Mar. 1995.

[6] P. Berardino, G. Fornaro, R. Lanari, and E. Sansosti, "A new algorithm for surface deformation monitoring based on small baseline differential SAR interferograms," IEEE Trans. Geosci. Remote Sensing, vol. 40, no. 11, pp. 2375-2383, Nov. 2002

[7] A. Reigber and A. Moreira, "First demonstration of airborne SAR tomography using multibaseline L-band data," IEEE Trans. Geosci. Remote Sensing, vol. 38, no. 5, pp. 2142-2152, Sept. 2000. 